\title{
Energy Consumption Patterns for Different Mobility Conditions in WSN
}

\author{
Manjusha Pandey, Shekhar Verma \\ Indian Institute of Information Technology, Allahabad, India \\ E-mail: \{rs58, sverma\}@iiita.ac.in \\ Received September 25, 2011; revised October 15, 2011; accepted November 18, 2011
}

\begin{abstract}
Wireless sensor networks are challenging networks regarding communication because of its resource constrained nature and dynamic network topology. Plenty of research has being going on throughout the world to optimize communication cost and overhead due to it in the ad hoc networks, thus efforts are being made to make the communications more energy efficient. The application spectrum and use cases of wireless sensor networks includes many critical applications as environmental monitoring, to resource monitoring, to Industrial measurements, to public safety applications and last but not the least to sensitive applications as military sector applications .The erratic size of such networks and along with its exotic topology pose a magnificent set of challenges to the routing algorithms designed and implemented within such networks. The present work concentrates on the comparative analysis of some of reactive and proactive protocols used in the wireless sensor networks. The parameter for comparative analysis is the energy consumption for different simulation time and for different mobility conditions based scenario.
\end{abstract}

Keywords: Sensor Networks, Battle Field Monitoring System, AODV, DYMO, LANMAR, OLSR

\section{Introduction}

A wireless sensor network [1] can be described as a wireless network comprising of a group of randomly deployed mobile communication nodes that interact among themselves without the need of use of any centralized authority or any fixed infrastructure. In these types of networks each node functions as a host (sender/receiver) as well as a router itself. Such networks have been considered with prime importance over the last decade because of the ever increasing demand for ubiquitous connectivity and emergence of new communication scenarios and applications. Some critical areas of applications of such networks being in the fields of military and civilian application as communication in the battle field, disaster management, vehicular movement or communication in traffic management and scientific exploration etc. In all these applications, group communication is more important. Multicasting provides necessary services for group communication in such applications. The most appreciated features of sensor networks include 1) Dynamic topology, 2) Bandwidth constrained links, 3) Energy constrained operation, and 4) Limited physical security. To enable the communication between two nodes in sensor networks it requires establishing a wirelesschannel (route) between them.

Generally, multi-hop routing is used as the nodes may or may not be within the wireless transmission range of one-another and thus highly depend on each other for forwarding of the data packets to the desired destination. There can be varied route selection criteria such as energy efficiency, bandwidth efficiency, low traffic control overheads etc. Since the topology of a sensor network is dynamic in nature and thus changes frequently, a routing protocol must be a distributed algorithm that computes multiple; cycle free routes while keeping the communication overhead to minimum and also to suit the specific needs, numerous routing protocols have been investigated proposed and implemented [1]. Routing in sensor network faces extreme challenges because of node mobility/dynamics, extremely large number of nodes and very limited communication resources (e.g. Bandwidth and energy).The other technological challenges sensor network includes communication stability, security, energy consumption and most importantly quality of services. For the experimental setup first the scenarios were created then the desired statistics were applied followed by the simulation and results were viewed and analyzed. 
We have considered the sensor network models in context of network simulations and to the best of our knowledge this is the only work that compares the energy consumption patterns of routing mechanisms for the detailed models on performance of sensor networks [2]. Figure 1 presents the flowchart of the implemented experimental setup.

The four different mobility conditions for the scenario discussed as follows illustrate the comparative analysis by using accurate and representative sensor network model. Though the present work considers the second scenario for the comparative analysis, the same could be extended for all the four scenarios implemented.

\section{State of Art}

There are many network optimization related issues of concern to be solved in WSNs as rate control, flow control, medium access control, congestion control, queue management, topology control and power control, etc. [3]. It is not easy to provide a complete overview with respect to all issues relating to network optimization in WSNs. But one of the most important issues is EnergyEfficient Routing Design because communication dominates the critical portion of energy consumption, routing design is considered to be core of sensor network design. Many routing algorithms have been proposed in prior research. The shortest path is fundamental consideration for network flow routing. A simple implementation of this consideration in sensor network is the minimum hop $(\mathrm{MH})$ routing. Many researchers have proposed and implemented shortest path algorithms so as to minimize the utilization of energy.

\section{Create Scenario for Battle Field Monitoring}

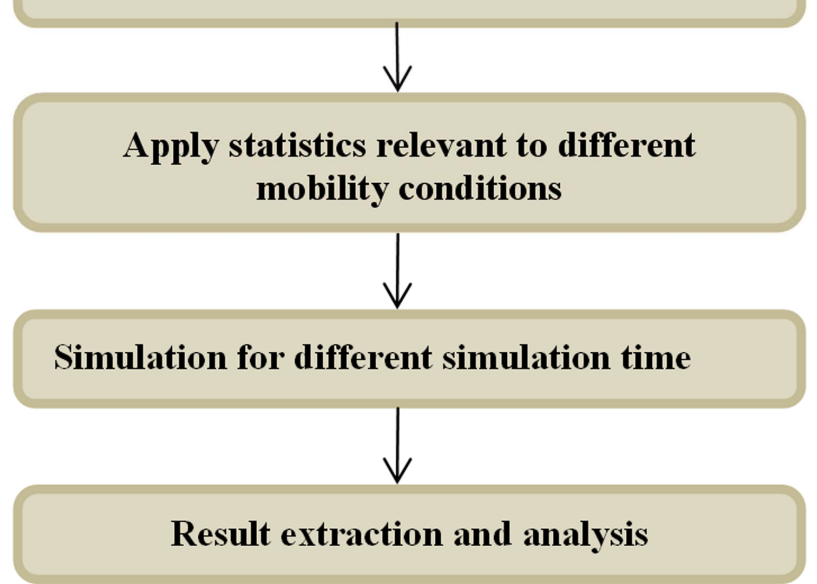

Figure 1. Flowchart for the simulation and implementation of the experimental setup.
Battery power is a very critical resource in sensor networks. This is particularly true for sensor networks which have been deployed in areas of low or poor accessibility. These sensor networks are expected to operate for longer periods without human intervention, ruling out the possibilities of replacing exhausted batteries. Such sensors need to be highly energy efficient to conserve battery power. Among other schemes, like choosing energy efficient routes, scheduling, data aggregation etc., sensors endeavors for the conservation of energy by periodically switching to a low energy consuming sleep state.When a node is not in sleep state, it is in another state termed as active state. Many routing techniques have been proposed but very few comparisons for different mobility conditions for different protocols have been done and analyzed. WSNs can be classified according to several aspects with impact on the routing protocol design. One such aspect is the mobility of nodes of the network and the base station. The nodes can be static or mobile. Considering the work done for performance comparisons of routing protocols the major focus have been on the evaluation based on quantitative and qualitative metrics. But the comparison of energy consumption of protocols for different exhaustive mobility conditions and different simulation intervals of the same scenario have not been proposed performed and analyzed yet. Various routing protocols used for the study are; AODV, DYMO, OLSR, and LANMAR.

In the proactive protocols OLSR [4], LANMAR, the nodes continuously searches for routing information in the network so that when a route is required, the route is known already. The routing information (distance vector or link state) of all the nodes is stored and updated in tabular forms at each node. Distance vector (DV) or linkstate (LS) route algorithms used in this routing protocol find shortest path to the destination.

OLSR [4] is a variation of traditional link state routing, modified for improved operation in ad hoc networks. The key feature of OLSR protocol is that it uses multipoint relays (MPRs) to reduce the overhead of network floods and size of link state updates. Each node maintains a route to every other node in the network. This technique significantly reduces the number of retransmissions in a flooding or broadcast procedure.

LANMAR combines the features of Fisheye State Routing (FSR) and Landmark routing. The key novelty is the use of landmarks for each set of nodes which move as a group (e.g., a team of co-workers at a convention or a tank battalion in the battlefield) in order to reduce routing update overhead. Like in FSR, nodes exchange link state only with their neighbors. Routes within Fisheye scope are accurate. Simulation experiments show that LANMAR provides efficient and scalable routing in 
large, mobile, ad hoc environments in which group mobility applies.

On the other hand, On-demand routing protocols like AODV [5], DYMO etc. are more dynamic. Instead of periodically updating the routing information, these pro tocols update routing information whenever a routing is required. This type of routing creates routes only when desired by the source node and therefore, in general, the signaling overhead is reduced compared to proactive approaches of routing.

DYMO is intended for use by mobile nodes in wireless, multi hop networks. DYMO determines unicast between DYMO routers within the network in an on-demand fashion, offering improved convergence in dynamic topologies. The basic operations of the DYMO protocol are route discovery (by route request and route reply) and route maintenance.

\section{Art WSN for Battle Field Monitoring System}

WSN for battle field monitoring System scenario demonstrates data collection from unattended ground sensors using mobile nodes. Sensors have been randomly deployed within the observation region. The sensors are constantly monitoring any phenomena which may be of interest in the area. The sensed information observed by any sensor is stored locally at the sensor itself. While the mobile vehicles move inside the area where sensors have been deployed. The vehicles are having short range of communication to the sensors and long distance communication to one remote site that is called fusion centre in the scenario. The sensors transmit their locally stored data to the vehicles that at any time are within their radio range.

The vehicles afterwards relay the sensed data packets to fusion centre with the help of long distance communication to that centre. Node types used in this scenario are: a) Ground Sensors (GS) which refers to ground sensors. b) Unmanned Vehicles (UV) which refers to mobile vehicles c) Fusion centre refers to a remote site. GS and UV are both battery-powered devices. Short rage communication between GSs and UVs has been configured using ZigBee. PHY and MAC protocol used in the scenario is 802.15.4 and the four protocols mentioned as the paper follows are used for the long distance communication between UVs and fusion centre. Fusion centre is configured as WiFi (802.11a) also different protocols have been used for this communication that have been defined by two communicating interfaces for the UVs and all the four routing protocols have been used in both the interfaces.

The scenario consists of: 100 GS nodes (nodes from 1 through 100) with linear battery model and micaZ radio energy model.5 UV (nodes from 100 through 105) with random way point mobility initially within the area where sensors are deployed (velocity range $0.1-0.4$ damp). Linear Battery model and micaZ radio energy model have been configured for GSs and UVs. Fusion center is the node 121. When the scenario is run, it shows that GVs moving within the area they are deployed.The UVs communicate with the other UVs that are within their ZigBee communication range. The sensors are having CBR flows to fusion centre and are able to send their sensed data to the fusion centre.

Figure 2 presents the framework for the battle field monitoring scenario implemented. The four different mobility conditions for the scenario considered may be described as follows:

1) All nodes within the network are static. The GS (ground sensors) and the UV(Unmanned station) are static in the first mobility condition considered while the fusion centre remains static in each and every mobility condition in which the scenario has been implemented.

2) In the second mobility condition for the scenario implemented is that the GS (ground stations) are static while the UV (unmanned Vehicle) are mobile.

3) The third mobility condition considered for the scenario says that the implementation of the GS (ground sensors) is mobile while the UV (unmanned Vehicles) are static.

4) The fourth mobility condition for which the scenario is implemented refers to the situation when the GS (ground sensors) are mobile as well as the UV (unmanned Vehicles) are also mobile.

The present work concentrates on the second senario descibed and the scenario has been tested for different simulation intervals in order to present a comparative

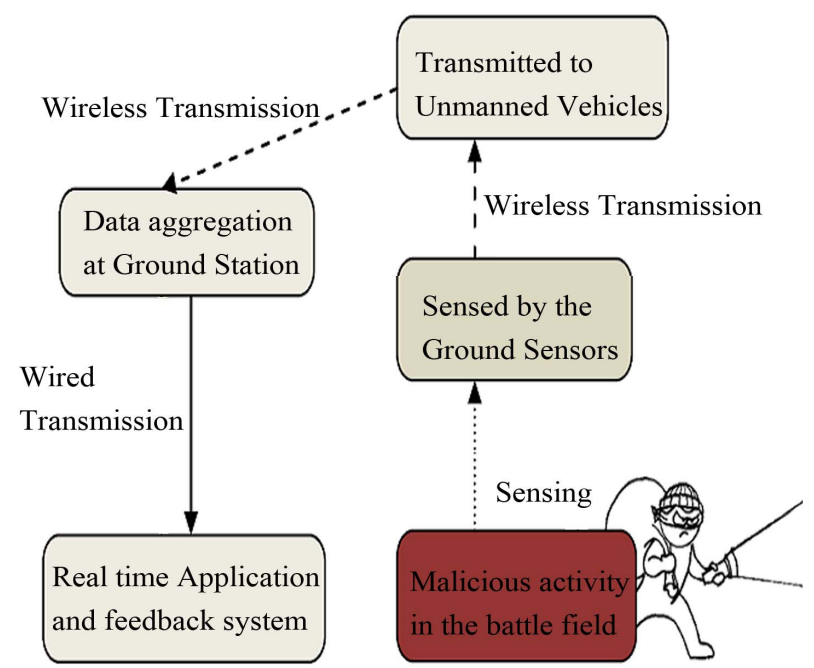

Figure 2. Framework of WSN for Battle field monitoring system. 
analysis of various routing protocol energy consumption pattern.

\section{Simulation Setup of WSN for Battle Field Monitoring System}

The overall objective of this simulation study is to analyze energy consumption and the performance of different existing wireless routing protocols designed and implemented in ad hoc wireless network environment. The simulations have been done using QualNet version 5 [6], a software that provides with the scalable simulations of Wireless Networks. In our simulation, we have a network of 100 nodes, having 5 tanks and 1 ground station placed randomly within a $500 \mathrm{~m} \times 500 \mathrm{~m}$ area and operating for over 30 seconds of the simulation time. Multiple executions with different seed numbers have been conducted for each scenario and collected data has been averaged over those runs. The two-ray propagation path loss model has been used in our experiments along with lognormal shadowing model. The parameters used for configuration of PHY802.15 of Ground Sensors (GS) and Unmanned Vehicles (UV).

The access scheme followed is CSMA/CA with acknowledgements. MAC layer parameters used are IEEE 802.15.4 for the ground sensors and IEEE802.1 for the unmanned vehicles and ground station or fusion center. The network layer affects the QoS if it has fewer queues, as it will queue packets of many a different service types into one queue that is priori queue. The node movements (except the ground sensor) in the experiments use the random waypoint mobility model with mobility speed ranging from $0 \mathrm{~m} / \mathrm{s}$ to $10 \mathrm{~m} / \mathrm{s}$. We choose this range because ad hoc wireless network support this medium mobility unlike static network [7].

\section{Simulation Parameters for Experimental Setup}

Experimental setup for the present comparative study was implemented for the following parameters:

Number of Nodes:

$\begin{array}{ll}\text { UGS } & 100 \\ \text { UGV } & 05 \\ \text { Ground station } & 01\end{array}$

Mac Parameters:

$\begin{array}{ll}\text { UGS } & \text { IEEE } 802.15 .4 \\ \text { UGV } & \text { IEEE } 802.11 \\ \text { Ground station } & \text { IEEE } 802.11\end{array}$

Ground station IEEE 802.11

Traffic Parameters: UGV

Data Payload

Path Loss Model

Mobility Model

CBR

1024 bytes/packet

Two Ray Model

Random Waypoint

Interface queue type
To evaluate the performance of routing protocols [8], both qualitative and quantitative metrics must be considered. Most of the routing protocols ensure the quantitative and qualitative metrics as the portion of packets sent by the application that are received by the receiver. The paper presents a exhaustive study of the energy consumed in sending and receiving modes by the nodes in the different mobility conditions. The study was further incorporated for different simulation duration for second scenario where the Ground Sensors (GS) are static Unmanned Vehicles (UV) are mobile.

Energy Consumption (mJoule): The MICAZ Mote devices are in the following four states: transmitting, receiving, idle and sleep. Energy consumption is the quantity of energy consumed by mote during the above mentioned states of the device. The unit of energy consumption used in the simulations is milliJoule [9].

Other Network Parameters:

$\begin{array}{ll}\text { Antenna } & \text { Omni directional } \\ \text { Simulation time } & 30 \mathrm{sec} \\ \text { Transmission range } & 35 \text { meter } \\ \text { Transmission Power(dbm) } & 3.0 \mathrm{dbm} \\ \text { Temperature } & 290.0 \\ \text { Node speed (mobility) } & \text { Min: } 0 \mathrm{~m} / \mathrm{sec} \text {; Max: } 10 \mathrm{~m} / \mathrm{sec} \\ \text { Area } & 500 \times 500 \text { meters } \\ \text { Energy Model } & \text { MICAZ } \\ \text { Battery Model } & \text { Simple Linear, } 1200 \mathrm{mAhr}\end{array}$

\section{Experimental Outcomes and Analysis}

The scenario under consideration for the consumption of energy in transmit mode for different simulation times is the second scenario where the ground sensors are static while the unmanned vehicles are mobile the following results were viewed for simulation times under consideration being 30 seconds, 300 seconds and 3000 seconds respectively for the reactive and proactive protocols under consideration.

Following figures presents the simulation results analyzed and concluded in detail. The first protocol implemented and analyzed is AODV.

1) AODV (Adhoc on Demand Distance Vector Routing)

AODV [5] routing uses the number of link hops as its routing metric. However since the limitation of battery power is one of the most essential concerns of sensor networks, routing algorithms for sensor networks attempt to optimize the consumption of this resource. Many researchers have proposed and implemented shortest path algorithms so as to minimize the utilization of energy. The results shown in the Figure $\mathbf{3}$ presents a linear increase that is directly proportional to the increase in the simulation interval from 30 seconds to 3000 seconds in the case of AODV routing protocol. 


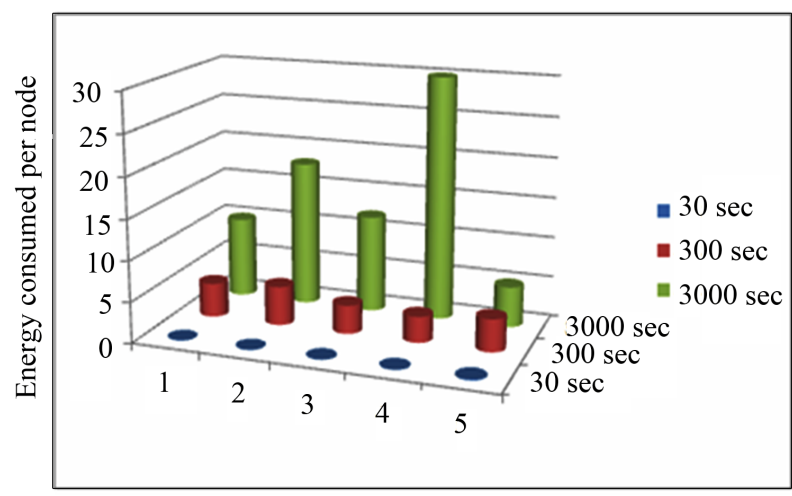

Unmanned Vehicles

Figure 3. Comparative energy consumption by AODV routing protocol for different simulation time interval of the second scenario.

\section{2) DYMO (Dynamic MANET On Demand Routing} Protocol)

On-demand routing protocols like AODV [10], DYMO [8] etc. are more dynamic. Rather than periodically up- dating the routing information, these protocols update routing information whenever a routing is required. DYMO is generally intended for the use by mobile nodes in wireless, and multi hop networks. DYMO determines unicast between DYMO routers within the network itself in on-demand fashion, which offers improved conve- rgence of the dynamic topologies. The basic operations defined in the DYMO protocol include route discovery (by route request and route reply) and route main- tenance. Though the results depicted in Figure 4 do not present a steep variation with increase in simulation time interval.

\section{3) OLSR (Optimized Link State Routing Protocol)}

OLSRa variation of traditional link state routing, has been modified for improved operation in ad hoc networks. The key characteristic of OLSR is that it makes use of multipoint relays (MPRs) that reduce the network floods overhead and thus the size of link state updates. The results present an immediate increase in the energy consumption with increasing simulation time interval from 300 seconds to 3000 seconds as shown in Figure 5.

4) LANMAR (Land Mark Routing Protocol)

LANMAR [11] adds up the features of Fisheye State Routing (FSR) and Landmark routing. The novelty of the protocol is the use of landmarks for every set of nodes which move as a group (e.g., a team of co-workers or a tank battalion of the battlefield) to reduce routing update overhead. Like the AODV routing protocol energy consumptions presents a steep increase with the increase in simulation time interval. Figure 6 gives a comparative view.

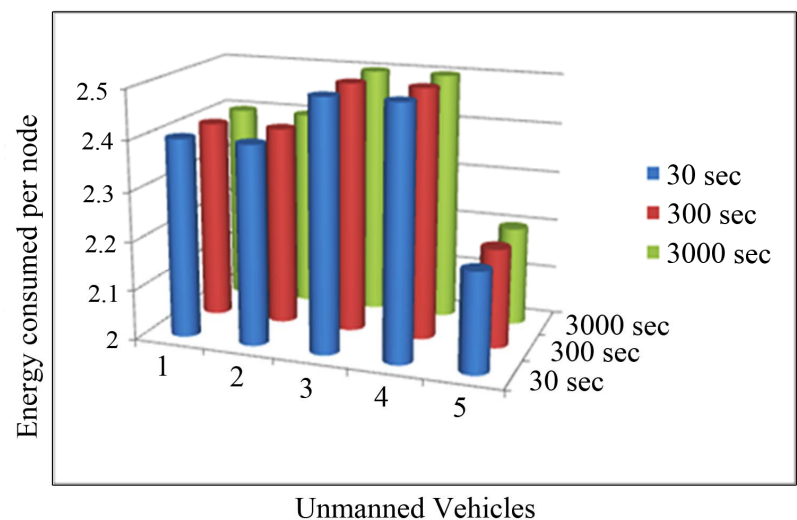

Figure 4. Comparative energy consumed by OLSR routing protocol for different simulation time for the second scenario.

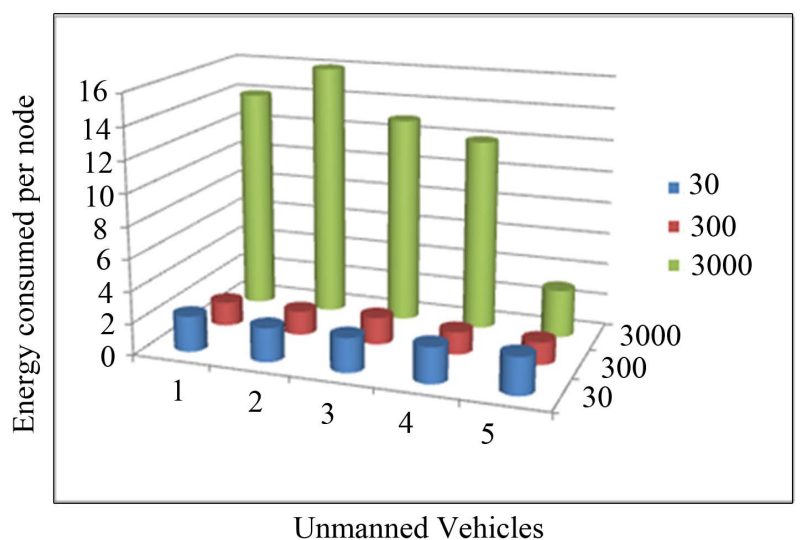

Figure 5. Comparative energy consumed by OLSR routing protocol for different simulation time for the second scenario.

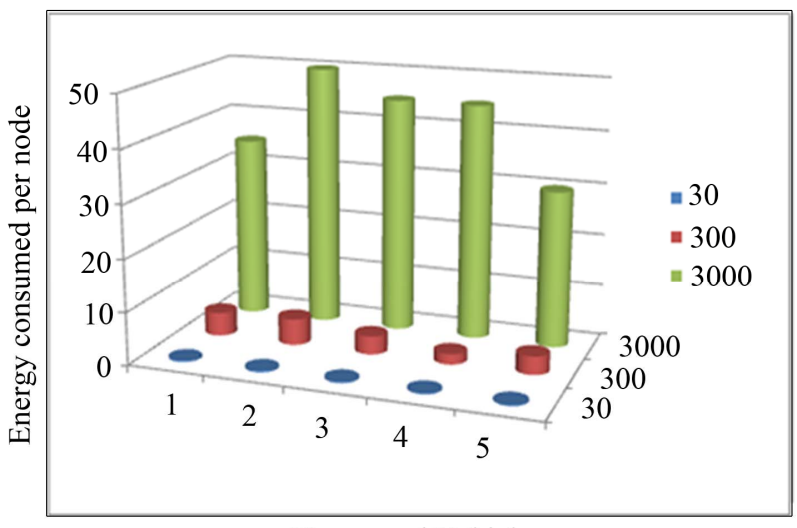

Unmanned Vehicles

Figure 6. Comparative energy consumed by OLSR routing protocol for different simulation time for the second scenario.

Simulation can be divided between less flexible but accurate simulation based approach and more generic but 
less detailed network simulator models. Simulator which provides a rich suite of following models: sensing stack to model wave and diffusion based sensor channels, an accurate battery model, processor power consumption model, energy consumption model and sensor network based traffic model [9].

The study could be used to present the effects of detailed modeling on the performance of higher layer protocols. Our results show that comparative analysis that was not affected by the changing scenario conditions with varied mobility conditions of the UV's and GS's As we know that the wireless sensor networks are resource constraint networks hence the performance of the routing protocols for the energy consumed may help for the selection of appropriate protocol. Though the selection of routing protocol in wireless sensor networks is also dependent on the applications for which the network may be used but the present results may always be helpful for the selection of energy efficient routing protocol.

\section{Conclusions}

The research area of ad hoc network [12] has attracted the academic world and the industry both due to its varied possible application for anytime, anywhere, any how communication scenario. This wide spectrum of applications possible for ad hoc networks has been made the network vividly applicable. The routing protocol for ad hoc networks has been a prime research domain during the present decade. Although extensive efforts have been exerted so for on the routing problem in wireless communications, there are still some challenges via multicasting that confront effective solutions to the routing problem. A number of such protocols have been developed. But none of the protocols has been found to be best suitable for all scenarios. All the protocols have their own advantages and disadvantages.

Depending on the constraints followed by the networks the routing algorithms have been updated and modified from time to time to make the routing more and more efficient and accurate. The present work proposes to find the effects of different patterns of node mobility within the network. The results though don't present a steep comparative orientation of the results towards a specific routing protocol but the comparative study leads towards some interesting results.

Further research is needed to find most suitable protocol for each and every scenario condition so that an optimized routing protocol could be suggested for various real life applications have concurrency to the mentioned scenarios of the simulated wireless network environment.

\section{References}

[1] C. Toh and E. Royer, "A Review of Current Routing Protocols for Ad Hoc Mobile Wireless Network," IEEE Personal Communications, Vol. 15, No. 5, 1999, pp. 46-55.

[2] I. F. Akyildiz, W. L. Su, Y. Sankarasubramaniam and E. Cayirci, “A Survey on Sensor Networks,” IEEE Communications Magazine, Vol. 40, No. 8, 2002, pp. 102-114. doi:10.1109/MCOM.2002.1024422

[3] S. Basagni, M. Conti, S. Giordano and I. Stojmenovic, “Mobile Ad Hoc Networking,” Wiley, Hoboken, 2004.

[4] C. Santivanez, B. McDonald, I. Stavrakakis and R. Ramanathan, "Making Link State Routing Scale for Ad Hoc Network," Proceedings of the 2nd ACM International Symposium On Mobile Ad Hoc Networking \& Computing, Long Beach, 4-5 October 2001, pp. 22-32.

[5] C. Perkins, E. B. Royer and S. Das, “Ad Hoc On-Demand Distance Vector (AODV) Routing,” Internet Draft, The Internet Society, Washington DC, 2003.

[6] Qualnet, http://www.scalablenetworks.com

[7] S. Basagni, M. Conti, S. Giordano and I. Stojmenovic, "Mobile Ad Hoc Networking," IEEE Press, Wiley, New York, 2004.

[8] S. Mohanty and S. K. Patra, "Prformance Analysis of Quality of Service Parameters for IEEE 802.15.4 Star Topology Using Manet Routing,” Proceedings of the International Conference and Workshop on Emerging Trends in Technology, Mumbai, 26-27 February 2010.

[9] W. Heinzeelman, B. Chandra A. Kasan and H. Bala Krishnan, "Energy-Efficient Communication Protocol for Wireless Micro-Sensor Networks," Proceeding of the 33rd Annual Hawaii International Conference on System Sciences, Maui, 4-7 January 2000, pp. 3005-3014.

[10] I. Chakeres and C. Perkins, "Dynamic MANET On-Demand (DYMO) Routing," IETF Internet Draft, The Internet Society, Washington DC, 2008.

[11] P. F. Tsuchiya, "The Landmark Hierarchy: A New Hierarchy for Routing in Very Large Networks," Computer Communication Review, Vol. 18, No. 4, 1988, pp. 35-42. doi:10.1145/52325.52329

[12] C. Siva Rama Murthy and B. S. Manoj, "Ad Hoc Wireless Networks: Architecture and Protocols,” 2nd Edition, Prentice Hall, Upper Saddle River, 2004. 